\title{
Infectious endocarditis with Coxiella Burnetii
}

\author{
Claudia-Elena Plesca ${ }^{1,2}$, Larisa Miftode², Ileana Traci², Isabela Ioana Loghin ${ }^{1,2}$, \\ Egidia Miftode ${ }^{1,2}$ \\ 1"Grigore T. Popa" University of Medicine and Pharmacy, lasi, Romania \\ ${ }^{2}$ Clinical Hospital for Infectious Diseases "St. Parascheva", Iasi, Romania
}

\begin{abstract}
$\mathrm{Q}$ fever is caused by an anthropozoonosis determined by the pathogen Coxiella burnetii, a gram-negative bacterium with intracellular growth. The occurrence of infection in the human species takes place through inhalation of contaminated aerosols or dust from infected domestic animals (cattle, sheep, goats) and more rarely through ingestion of unpasteurized milk, infected mite or inter-human transmission. The endocardium is one of the main infection sites, especially in the context of the long-term development of the disease, and cardiac decompensation often leads to death in absence of a proper diagnosis and appropriate treatment (1). We present the case of a patient of the male sex aged 37 years without personal pathologic history known admitted in "St. Parascheva" Clinical Hospital for Infectious Diseases lasi complaining of productive cough, fatigue, shortness of breath with moderate effort and pain in the left scapulohumeral joint with irradiation in the left upper limb. Clinical examination objectified digital clubbing, systolic/diastolic murmurs throughout the precordium area and hepato-splenomegaly, while laboratory tests revealed the presence of inflammatory syndrome, cholestasis and hepatic cytolysis. Echocardiography shows a hyperechogenic entity at the level of the aortic valve, as well as a severe valve disorder.

The diagnosis of infective endocarditis is established on aortic valve and therapy with first-choice antibiotics, consisting of triple combination of cefotaxime, amikacin and vancomycin, is initiated. Blood cultures taken upon admission were negative, while positive serological phase I and II tests for $C$. burnetii urged the indication of changing therapy with doxycycline and trimethoprim sulfamethoxazole (in the absence of hydroxychloroquine). Subsequently he underwent aortic valve replacement. The particularities of this case consisted in atypical clinical manifestations, the absence of fever and epidemiological context suggestive for $Q$ fever.
\end{abstract}

Keywords: Q fever, infectious endocarditis, valvulopathy

Q fever, also known as Derrick- Burnet disease, represents an infectious disease whose etiologic agent is Coxiella burnetii, a gram-negative intracellular coccobacillus with different morphological forms, variable virulence (phase I - virulent and immunogenic, phase II - much tempered) and increased resistance in the external environment $(1,2)$. Transmission to humans is through inhaling contaminated aerosols, multiplying in the lungs and dissemination into blood $(1,3)$. Infection may evolve without visible signs or various manifestations when generically called Q fever (from the
Engl. query: question, disease with initially unknown etiology) (4).

Q fever is a widespread zoonosis, source of infection being represented by domestic animals (sheep, horses, dogs, cats), rarely birds and rodents $(2,5)$. Due to the way of transmission, the greatest exposure to infection with Coxiella burnetii have people with certain occupations involving contact with potentially diseased animals: veterinarians, farmers, workers in the meat-processing industry or in slaughterhouses (4.5). Chronic form can manifest as endocarditis and is common in people with 
pre-existing valve disorders, valvular prostheses or immunodepression $(3,6)$. Q fever incidence is globally uniform, being widespread, with few exceptions (the Antarctic and New Zealand), on the territory of the majority of world states $(4,5,6)$.

Clinical manifestations occurring within the acute infection with $C$. burnetii varies from absence of symptoms, but with seroconversion (50\%-90\% of patients) to the stage of severe disease with multiorgan involvement $(2,5,6)$.

\section{CASE REPORT}

Patient P.C., male, aged 37 years, coming from urban areas, is admitted in May 2017 in the Clinical Hospital for Infectious Diseases in Iasi presenting productive cough with hemoptoic sputum, moderate physical asthenia with sweats and pain the left forearm and left scapulohumeral joint, symptoms which first occurred approximately two months prior to hospital admission, to which was added shortness of breath to the moderate efforts, with a more recent onset of about 2 weeks.

Objective clinical examination has shown the absence of fever, sclero-tegumental pallor, the presence of digital clubbing, systolic/diastolic murmurs grade IV/VI, audible in all areas of cardiac auscultation, but with maximum intensity in the aortic and mitral areas, liver at $2 \mathrm{~cm}$ below the costal margin and splenomegaly grade I.

Laboratory explorations at hospitalization showed the following pathological changes: biological syndrome of intense inflammation, intense syndrome of cholestasis and moderate liver cytolysis without major changes in blood culture (Table 1).

TABLE 1. Laboratory markers evolution

\begin{tabular}{|l|l|l|l|l|}
\hline & Day 1 & Day 7 & Day 12 & Day 19 \\
\hline WBC/mm ${ }^{3}$ & 6,080 & 4,270 & 4,510 & \\
\hline NEUT\% & 45.7 & 49.4 & 48.5 & \\
\hline RBC*10 $/ \mathrm{mm}^{3}$ & 5.4 & 5.1 & 5.2 & \\
\hline HGB g/de & 13.9 & 13.6 & 13.8 & \\
\hline $\mathrm{PLT}\left({ }^{*} 10^{3} / \mathrm{mm}^{3}\right)$ & 203 & 182 & 219 & \\
\hline ALT UI/I & $\mathbf{1 2 1}$ & $\mathbf{2 3 0}$ & $\mathbf{2 1 0}$ & $\mathbf{1 0 6}$ \\
\hline AST UI/I & $\mathbf{6 3}$ & $\mathbf{1 3 2}$ & $\mathbf{1 1 2}$ & $\mathbf{8 0}$ \\
\hline bilirubin $\mathrm{mg} / \mathrm{dl}$ & $\mathbf{1 . 1 8}$ & 1.01 & $\mathbf{1 . 3 8}$ & 0.89 \\
\hline $\begin{array}{l}\text { alkaline } \\
\text { phosphatase UI/I }\end{array}$ & $\mathbf{7 4 9}$ & - & $\mathbf{8 3 4}$ & - \\
\hline urea & 46 & 35 & 33 & 46 \\
\hline crea nine & 0.88 & 1.04 & 0.98 & 1.17 \\
\hline ESR $\mathrm{mm} / \mathrm{h}$ & $\mathbf{6 0}$ & $\mathbf{6 0}$ & $\mathbf{1 5}$ & - \\
\hline CRP $\mathrm{mg} / \mathrm{I}$ & - & - & - & $\mathbf{9 . 0 8}$ \\
\hline
\end{tabular}

Electrocardiogram (EKG) showed the presence of a minor right bundle branch (RBBB), while transthoracic echocardiography (TTE) described a hyperecogenic entity at the level of the aortic valve (attached to the antero-lateral aortic cusp), with size of 30/18 mm and synchronous movements. The echocardiographic exam also objectified the presence of severe bicuspid aortic valve and aortic valve stenosis.

Abdominal ultrasound showed an enlarged liver, with a right lobe of $160 \mathrm{~mm}$, with normal ecogenicity, main biliary ducts, intrahepatic biliary ducts, portal vein of normal size; gall bladder moderately enlarged, septate, without gallstones; spleen measuring $131 \mathrm{~mm}$; pancreas, kidneys without structural pathologic changes.

At the time of admission, 3 blood cultures were collected and empirical therapy with antibiotics (amikacin, cefotaxime and vancomycin) was initiated. The clinical evolution of the patient remained stationary, and biological markers of inflammation have seen an uptrend during the following 12 days of hospitalization, as is evident from the data presented in table 1.

On the tenth day following admission, serological results for Coxiella burnetii were obtained and proved to be intensely positive (phase I Ig G antibodies as well as phase II Ig G antibodies) (Table 2).

TABLE 2. Serological markers for $Q$ fever

\begin{tabular}{|l|l|l|}
\hline Serum & Result & Ref. Range \\
\hline Q-Fever Phase 1 IgG Ab & positive & \\
\hline Q-Fever Phase 1 IgA Ab & nega ve & \\
\hline Q-Fever Phase 2 IgG Ab & $467 \mathrm{UI} / \mathrm{ml}$ & nega ve $<20$ \\
\hline & & posi ve $>30$ \\
\hline Q-Fever Phase 2 IgM Ab & nega v & \\
\hline & $\begin{array}{l}\text { phase 1 antibodies detected! Se- } \\
\text { rological findings like cronic in- } \\
\text { fection with Coxiella burnetii }\end{array}$ \\
\hline
\end{tabular}

Corroborating clinical and laboratory data with positive serology for C. burnetii led to establishing the diagnosis of infective endocarditis on the aortic valve caused by Coxiella burnetii, so that therapy with antibiotics was reshuffled with doxycycline and co-trimoxazole (in the absence of hydroxicloroquine). Further evolution was favourable, with remission of symptoms and regression of inflammatory syndrome, cholestasis and liver cytolysis syndrome. The patient was transferred to the County Emergency Hospital with the recommendation 
to continue therapy with antibiotics orally for a period of at least 12 months, and to repeat the serological investigations. The patient subsequently underwent surgery with favourable postoperative evolution.

\section{DISCUSSION}

Q fever represents an infectious pathology with universal spread. In the acute form, prolonged fever may occur, acute hepatitis, atypical pneumonia, but over $60 \%$ of cases are asymptomatic $(1,2,7)$. In Romania, the number of cases diagnosed with $\mathrm{Q}$ fever is reduced (6 cases in 2007) in comparison with other European countries (8).

The most important risk factors for the progression of infection with Coxiella burnetii towards endocarditis are age (average age under 50 years) and valvular heart disease $(4,8)$. The main risk factor in the development of endocarditis in patients with $\mathrm{Q}$ fever represents significant valvular damage, but such a development was also registered in patients without a previous diagnosis of valvular disorder or with minor and silent valvular disorders. For these reasons, all persons recently diagnosed with $\mathrm{Q}$ fever must have an echocardiographic examination to exclude infectious endocardial process (9).

Infectious endocarditis incidence occurring in the context of acute infection with $C$. burnetii commonly associated with increased mortality in the absence of specific therapy varies from $0.6 \%$ to $7 \%$, depending on the epidemiological context $(2,10)$. Although the vast majority of cases are subclinical, it was observed that symptomatic forms almost always associate with endocardial lesions, regardless of bacterial genotype identified $(5,7,11)$. A number of large-scale studies have shown that Q fever endocarditis can lead to death in a proportion of $5 \%-65 \%$ of the cases, may require surgery in
$15 \%$ to $73 \%$ of the cases, or may evolve in the form of multiple relapses under inappropriate antibiotic treatment (13).

Due to the fact that, most often, the diagnosis of $Q$ fever precedes the one of infectious endocarditis, some researchers have demonstrated the effectiveness of prophylactic antibiotherapy administered for a period of 12 months in the case of patients with pre-existing valvular heart disease, as well as the usefulness of serological testing at 3 and respectively 6 months $(9,13)$.

In the case of chronic Q fever, a systemic inflammatory syndrome is most often present, with hepato-splenomegaly, absence of fever (atypical cases), which contributes to delaying the diagnosis and specific treatment (2.13). In a study published in 2013, Million and collaborators have noted a higher frequency of endocarditis with $C$. burnetii infections in male patients (71\%) and at a younger age (average age under 50 years of age) $(11,14)$.

\section{CONCLUSIONS}

Systemic manifestations of chronic Q fever may constitute important clues in diagnosing infection with Coxiella burnetii. Patients with valvulopathies or immunodepression with negative blood cultures must be taken into account for the exclusion of this condition, even if they do not come from endemic areas or professions at risk. The correct and quick diagnosis of $\mathrm{Q}$ fever can be determined by carrying out serological tests, and the choice of targeted antibiotic therapy with the appropriate length is the only therapeutic alternative in such cases. The particularity of the presented case consists in the absence of fever throughout the evolving disease, poorly expressed symptoms and the absence of meaningful epidemiological and professional context.

Conflict of interest: none declared Financial support: none declared

\section{REFERENCES}

1. Voth D.E., Heinzen R.A. Lounging in a lysosome: the intracellular lifestyle of Coxiella burnetii. Cell Microbiol. Apr 2007; 9(4): 829-40

2. Carole Eldina et al. From $Q$ Fever to Coxiella burnetii Infection: a Paradigm Change. Clin. Microbiol. J. January 2017; 30(1); 115-190.

3. Deyell M., Brian C., Ross D., Alvarez N. Q fever endocarditis: A case report and review of the literature. Can J Cardiol. 2006; 22(9): $781-785$.

4. Barten D.G. et al. Localizing chronic $Q$ fever: A challenging query. BMC Infect Dis. 2013; 13: 413.

5. Million M., Thuny F., Richet H., Raoult D. Long term outcome of $Q$ fever endocarditis: A 26 year personal survey, Lancet Infect Dis, 2010; 10; 527-535.

6. Mogollon M.V., Anguita M.P., Aguado J.M. et al. $Q$ fever endocarditis in Spain clinical characteristics and outcome. Enferm Infec Microbiol Clin, 2011; 29; 109-116. 
7. lung B., Baron G., Tornos P., Gohlke-Barwolf C., Butchart E.G., Vahanian A. Valvular heart disease in the community: A European experience. Curr Probl Cardiol, 2007; 32; 609-661.

8. Edouard S., Mahamat A., Demarr M., Abboud P., Djossou F., Raoult D. A conparison between emerging $Q$ fever in French Guiana and endemic Q fever in Marseille France, Am J Trop Med Hyg, 2013; 29;133-146.

9. Glazunova O., Roux V., Freylikman O. et al. Coxiella burnetii genotyping, Emerg Infect Dis, 2005; 11; 1211-1217.

10. Hartzell J.D., Gleeson T., Scoville S., Massung R.F., Wortmann G., Martin G.J. Practice guidelines for the diagnosis and management of patients with $Q$ fever by the Armed Forces Infectious Diseases Society, Mil Med, 2012; 177; 484-494.

11. Million M., Walter G., Thuny F., Habib G., Raoult D. Evolution from acute $Q$ fever to endocarditis is associated with underlying valvulopathy and age and can be prevented by prolonged antibiotic treatment, 2013; Clin Infect Dis; 57 (6); 836-844.

12. Duval X., Alla F., Hoen B. et al. Estimated risk of endocarditis in adults with predisposing cardiac conditions undergoing dental procedures with or without antibiotic prophylaxis, Clin Infect Dis, 2006; 42; 102-107.

13. Kampschreur L.M., Dekker S., Hagenaars J.C. et al. Identification for risk factors for chronic $Q$ fever, the Netherlands, Emerg Infect Dis, 2012; 18; 563-570.

14. Lancelotti P., Tribouilloy C., Hagendorff A. et al. European Association Echocardiography recommendation for the assessment of valvular regurgitation (native valve disease), Eur J Ecocardiogr I, 2010; 11; 223-244. 